\title{
Endowed Multi-functionality of Branched Oligosaccharide from Corn Starch by Phosphorylation and Oleylation
}

\author{
(Received December 6, 2010; Accepted February 28, 2011)
}

(J-STAGE Advance Published Date: April 25, 2011)

\author{
Kenta Hagiwara, ${ }^{1}$ Reika Ogiwara, ${ }^{1}$ Makoto Hattori, ${ }^{1}$ Tadashi Yoshida, ${ }^{1}$ \\ Masatsugu Yamashita ${ }^{2}$ and Koji Takahashi ${ }^{1, *}$ \\ ${ }^{1}$ Department of Applied Biological Science, Faculty of Agriculture, Tokyo University of Agriculture and Technology \\ (3-5-8 Saiwai-cho, Fuchu, Tokyo 183-8509, Japan) \\ ${ }^{2}$ Quality Assurance Division, Taiyo Kagaku Co. \\ (1-3-1 Takaramachi, Yokkaichi, Mie 510-0844, Japan)
}

\begin{abstract}
Oleyl-branched oligosaccharide phosphate (OA-BOS-P) was prepared by phosphorylation through dry-heating branched oligosaccharide (BOS) from corn starch with metaphosphric acid, and then by oleylating the resulting branched oligosaccharide phosphate (BOS-P) through lipase-catalyzed solid phase synthesis. The multi-functionality of OA-BOS-P was evaluated in respect of its interfacial ability, $\mathrm{Ca}^{2+}$-binding ability, and ability to control the gelatinization and retrogradation behavior of potato starch. OA-BOS-P exhibited markedly lower surface tension and interfacial tension than either BOS-P or oleyl BOS (OA-BOS), and revealed $\mathrm{Ca}^{2+}$-binding ability similar to that of BOS-P. OA-BOS-P offered improved gelatinization behavior, as indicated by the elevated gelatinization temperature, reduced enthalpy, and reduced peak viscosity and breakdown, in comparison with those properties of other related samples. OABOS-P also inhibited retrogradation as indicated by the reduced setback viscosity, turbidity and development of an ordered structure depending on the level of addition, whereas BOS-P and OA-BOS elevated the turbidity, in spite of the reduced setback value and development of an ordered structure. OA-BOS-P could therefore be a useful multi-functional food material with interfacial, $\mathrm{Ca}^{2+}$-binding, and starchy foodcontrolling abilities.
\end{abstract}

Key words: acylated branched oligosaccharide phosphate, branched oligosaccharide phosphate, interfacial ability, $\mathrm{Ca}^{2+}$-binding ability, improved gelatinization behavior, inhibited retrogradation behavior

Controlling the stabilization of lipids, bioavailability of dietary calcium, and such thermal properties as the gelatinization and retrogradation of starch is regarded as important, because most foods contain oil and fat, a calcium-absorption inhibitor and starch. Such importance has encouraged the development of emulsifiers such as sucrose fatty acid ester (SE), ${ }^{1)}$ such $\mathrm{Ca}^{2+}$-binding materials as alginic acid, ${ }^{2)}$ casein phosphopeptide, ${ }^{3)}$ phosphorylated oligosaccharide ${ }^{4,5)}$ and citric acid, ${ }^{6}$ ) and such starchy food-modifying materials as SE, ${ }^{7)}$ monoacylglycerol ${ }^{8)}$ and related lipids. ${ }^{9)}$ In particular, since starch functions as a builder or modifier of starchy food, considerable attention has been focused on controlling the physical properties of starch. Starch reveals its characteristic viscoelasiticity only when heated in the presence of water, and the swollen starch granules easily break down under shear stress that often causes the disliked features of sticki-

\footnotetext{
* Corresponding author (Tel. +81-42-367-5712, Fax. +81-42-360-8830, E-mail:k-taka@cc.tuat.ac.jp)

Abbreviations: BOS, branched oligosaccharide; BOS-P, branched oligosaccharide phosphate; OA-BOS, oleyl-branched oligosaccharide; OA-BOS-P, oleyl-branched oligosaccharide phosphate; SE, sucrose fatty acid ester; SE-P, phosphorylated sucrose fatty acid ester; POS, phosphorylated oligosaccharide; PS, potato starch; DP, degree of polymerization; DSC, differential scanning calorimetry; RVA, rapid viscoanalyzer; PV, peak viscosity; $\mathrm{BD}$, breakdown; $\mathrm{SB}$, setback; MPA, metaphosphoric acid.
}

ness and a pasty texture. The resulting paste then deteriorates in its physical properties during subsequent cooling, degrading the quality of starchy food. Food processing often requires a food material with both specified and diverse functions, making it important to evaluate the characteristic functions of a food material from diverse viewpoints, and to develop new multi-functional food materials.

We have previously reported that phosphorylated sucrose fatty acid ester (SE-P), called an ionic glycolipid, could be prepared by dry-heating SE with metaphosphoric acid. SE-P was a multi-functional material that could improve the emulsifying ability of SE that was lost in the acidic region and presence of salt, could endow $\mathrm{Ca}^{2+}$-binding ability, and could modify the physical properties of starch in terms of reduced viscosity and depressed retrogradation. ${ }^{10)}$ However, SE-P is restricted by its relatively low solubility at low temperature, and by its increased gelatinization temperature and reduced viscosity. Improving the latter two properties by enhancing the thermal stability of starch could help to inhibit the development of a sticky or pasty texture. A larger saccharide moiety in an ionic glycolipid would bring about both improved solubility due to its greater hydration and also stabilization of starch; this would result from reduced motion of the starch chains due to stabilization of the water structure surrounding the starch chains by interaction among starch, water and the 
saccharide moiety. ${ }^{11,12)}$

Phosphorylated oligosaccharides (POS) with $\mathrm{Ca}^{2+}$-binding ability have recently been developed from potato starch by thoroughly digestion with $\alpha$-amylase, glucoamylase and pullulanase as a mixture of mono- and di-phosphoryl $\alpha-1,4-$ linked maltooligosaccharides with an average degree of polymerization $(\overline{\mathrm{DP}})$ of $4.02{ }^{4,5)}$ However, these POS had no emulsifying ability because of the lack of a hydrophobic moiety, and the yield was not elucidated; the lack of information may have been due to the very low yield resulting from the low phosphate content of the original potato starch used. When digesting corn starch with $\alpha$-amylase and $\beta$-amylase to produce a sweetener, a certain amount of branched oligosaccharide (BOS), being larger and more bulky than POS, is invariably obtained as a by-product and can be used as a food material. However, BOS does not have $\mathrm{Ca}^{2+}$-binding ability or interfacial ability. A new BOS-based multi-function food material is therefore needed that could be developed by applying such treatments as phosphorylation and fatty acylation.

Fatty-acylating phosphorylated BOS (BOS-P) by a mild procedure such as lipase-catalyzed solid-phase synthesis, which has endowed sericin with good emulsifying ability, ${ }^{13)}$ should enable a new anionic glycolipid to be developed with $\mathrm{Ca}^{2+}$-binding ability and emulsifying ability. In addition, acylated BOS-P would have high solubility and outstanding ability to control the thermal properties of starch, because it has a more bulky hydrophilic saccharide moiety than either SE-P or POS. A saturated fatty acid can more preferentially form a complex with a starch chain than an unsaturated fatty acid. ${ }^{14,15)}$ However, an unsaturated fatty acid such as oleic acid has the advantage of easier handling than a saturated fatty acid such as stearic acid because of its lower melting temperature.

Olelyl BOS-P (OA-BOS-P) was prepared in this study from BOS by phosphorylating BOS through dry-heating with metaphosphoric acid, and by subsequently oleylating the resulting BOS-P through lipase-catalyzed solid phase synthesis. The multi-functional properties of OA-BOS-P were demonstrated by the interfacial ability, $\mathrm{Ca}^{2+}$-binding ability and starch-modifying ability in terms of the elevated gelatinization temperature, reduced viscosity and inhibited retrogradation; this was indicated by the reduced setback viscosity and turbidity, and development of an ordered structure for potato starch.

\section{MATERIALS AND METHODS}

Materials. BOS was supplied by Sanmatsu (Tokyo, Japan), having been prepared by rapidly heating corn starch to $105^{\circ} \mathrm{C}$ to react with heat-resisting $\alpha$-amylase at $95^{\circ} \mathrm{C}$, before being thoroughly digested with $\beta$-amylase after cooling to $55^{\circ} \mathrm{C}$. The reaction product was applied to size-exclusion chromatography and ion-exchange chromatography before being decolorized with active charcoal, and then spray-dried to obtain BOS. An immobilized lipase (Novozym 435; Candida antartica, 10,000 PLU/g) was purchased from Novozyme Japan (Tokyo, Japan), and acid phosphatase (potato, $2 \mathrm{U} / \mathrm{mg}$ ) was purchased from Roche Diagnostics (Basel, Switzerland). Large granules of potato starch (PS; Hokuren Research Institute, Sapporo, Japan) were used after being repeatedly washed with distilled water at $4{ }^{\circ} \mathrm{C}$ and air-dried (15.3\% residual moisture in the recovered starch). Sucrose mono-oleiate (O-1570) and mono-stearate (S-1570) were obtained from Mitsubishi-Kagaku Foods Co. (Tokyo, Japan). All other reagents used were commercially available.

Preparation of phosphorylated BOS (BOS-P). BOS was phosphrylated by dry-heating with metaphosphoric acid (MPA) according to the method of Yamagishi et al. ${ }^{10)}$ that was based on the method of Tarelli and Wheeler. ${ }^{16)}$ In brief, BOS $(10 \mathrm{~g})$ was dissolved in $100 \mathrm{~mL}$ of water, and an MPA solution $(62 \mathrm{~g} / 100 \mathrm{~mL}$, adjusted to $\mathrm{pH} 5.5$ with $5 \mathrm{M} \mathrm{NaOH})$ was added (BOS:-OH group of MPA $=1: 1.5$, molar ratio). The reaction mixture was lyophilized, and then heated at $110^{\circ} \mathrm{C}$ for $15 \mathrm{~h}$ to be phosphorylated. The reaction product was dissolved in $5.1 \mathrm{~L}$ of water, and then un-reacted MPA was electrodialyzed by a TS-2-10 instrument (Tokumaya, Tokyo, Japan) equipped with an AC-220 desalting cartridge (Astom, Shizuoka, Japan) to give $2 \mathrm{mS} / \mathrm{cm}$ as described previously. ${ }^{17)}$ The resulting dialysate was adjusted to $\mathrm{pH} 5.5$ by adding a one-tenth volume of a $0.2 \mathrm{M}$ acetate buffer ( $\mathrm{pH}$ 5.5), and then applied to a DEAE-Sepharose fast flow column (2.8 i.d. $\times 57 \mathrm{~cm}$ : Amersham Pharmacia Japan, Tokyo, Japan). The adsorbed fraction was eluted with a $0.02 \mathrm{M}$ acetate buffer ( $\mathrm{pH} 5.5$ ) containing $1.0 \mathrm{M} \mathrm{NaCl}$ at a flow rate of $5 \mathrm{~mL} / \mathrm{min}$. The eluate was similarly electrodialyzed to give $1 \mathrm{mS} / \mathrm{cm}$, and lyophilized to recover BOS-P.

Preparation of olelyl BOS-P (OA-BOS-P). BOS-P was oleylated according to the method previously described. ${ }^{13)}$ In brief, $500 \mathrm{mg}$ of BOS-P in a screw-capped Erlenmeyer flask was pulverized in $50 \mathrm{~mL}$ of dehydrated $n$-hexane by a Polytron PTA-7 homogenizer (Kinematica, Switzerland) at $24,000 \mathrm{rpm}$ for $1 \mathrm{~min}$. Molecular sieves $(0.5 \mathrm{~g})$ activated at $500^{\circ} \mathrm{C}$ for $2 \mathrm{~h}$ were added, and the mixture was de-aerated under reduced pressure for $1 \mathrm{~min}$ at room temperature. Oleic acid $(5.0 \mathrm{~mL})$ and $5 \mathrm{~g}$ of Novozym 435 were then added, and the headspace gas was replaced with nitrogen gas. The reaction mixture was incubated at $60^{\circ} \mathrm{C}$ for $24 \mathrm{~h}$ while shaking at $60 \mathrm{cycle} / \mathrm{min}$. The reaction product was passed through a stainless steel mesh to remove the molecular sieves, and then passed through a membrane filter $(0.2-\mu \mathrm{m}$ pore size; Advantec, Tokyo, Japan) to recover the residue. This residue was dispersed in chloroform $/ n$-hexane $(5: 1, \mathrm{v} / \mathrm{v})$, and the dispersion was centrifuged at $1,700 \times \mathrm{G}$ for $5 \mathrm{~min}$ to separate the added Novozym 435 rising to the surface of the solvent. The recovered Novozym 435 was washed twice with $n$-hexane and then twice with ethanol. The reaction product adhering to Novozym 435 that had been collected by filtering at reduced pressure was extracted by adding a small amount of chilled water. The filtrate was lyophilized to recover OABOA-P. Oleyl BOS (OA-BOS) was similarly prepared, except for recovering Novozym 435 as a precipitate by using a dispersing solvent of chloroform $/ n$-hexane $(2: 1, \mathrm{v} / \mathrm{v})$.

Measurement of the unit chain-length distribution of BOS.

BOS $(10 \mathrm{mg})$ dissolved in $2 \mathrm{~mL}$ of a $0.2 \mathrm{M}$ acetate buffer ( $\mathrm{pH} 4.8$ ) was completely de-branched with isoamylase (26 IU) and pullulanase (170 IU) according to the previous method. ${ }^{18)}$ The de-branched sample solution was thoroughly dialyzed against distilled water by using a Spectra/Por dialyzing membrane (100 molecular weight cutoff; Medical Industry, Breda, The Netherlands), before being lyophilized to recover the de-branched sample. The de-branched sample 
was dissolved in $75 \%$ dimethyl sulfoxide $(4.6 \mathrm{mg} / 0.8 \mathrm{~mL})$. The sample solution was loaded into a Dionex high-performance anion-exchange chromatograph (HPAEC) coupled with a Dionex Carbopac PA guard column (3.0 i.d. $\times 25$ $\mathrm{mm}$ ), a Carbopac PA1 separation column (4.0 i.d. $\times 250$ $\mathrm{mm}$ ), and a pulsed amperometric detector (PAD) (Dionex Japan, Tokyo, Japan), and eluted by a linear gradient from $0.1 \mathrm{M} \mathrm{NaOH}$ to $0.1 \mathrm{M} \mathrm{NaOH}$ containing $0.6 \mathrm{M}$ sodium acetate at a flow rate $1.0 \mathrm{~mL} / \mathrm{min}$.

Differential scanning calorimetry (DSC). A BOS, BOSP, OA-BOA, or OA-BOA-P solution $(10 \mu \mathrm{L})$ containing 3, 5 and $10 \% \mathrm{w} / \mathrm{w}$ of each based on the dry starch weight, which had been adjusted to $\mathrm{pH} 7.0$ with $0.1 \mathrm{M} \mathrm{NaOH}$, was added to $5 \mathrm{mg}$ of PS in an air-tight anodized aluminum capsule. DSC was conducted to determine the gelatinization temperature and enthalpy change of gelatinization in the range of $5-100^{\circ} \mathrm{C}$ at a heating rate of $2 \mathrm{~K} / \mathrm{min}$ by using an SSC-5020 DSC6100 instrument (SII NanoTechnologies, Chiba, Japan) as previously described. ${ }^{19)}$ Distilled water $(15 \mu \mathrm{L})$ was used as a reference, and triplicate measurements were performed. DSC for the starch samples preserved at $4{ }^{\circ} \mathrm{C}$ for 7 days was also carried out to evaluate the re-gelatinization enthalpy as an index for relatively long-term retrogradation.

Viscosity measurement. After adding $25 \mathrm{~mL}$ of a BOS, BOS-P, OA-BOA, or OA-BOA-P solution $(10 \mu \mathrm{L})$ containing 0.5 and $1.0 \% \mathrm{w} / \mathrm{w}$ of each based on the dry starch weight, which had been adjusted to $\mathrm{pH} 7.0$ with $0.1 \mathrm{M} \mathrm{NaOH}$, to $2.0 \mathrm{~g}$ of PS in an aluminum container, an RVA Super 3 Rapid Viscoanalyzer $^{\mathrm{TM}}$ (Newport Scientific, NSW, Australia) was used to investigate the pasting properties. Each sample was held at $50^{\circ} \mathrm{C}$ for $1 \mathrm{~min}$, and then heated to $95^{\circ} \mathrm{C}$ at a heating rate of $5.6 \mathrm{~K} / \mathrm{min}$. After holding the temperature at $95^{\circ} \mathrm{C}$ for $2 \mathrm{~min}$, the sample was cooled to $50^{\circ} \mathrm{C}$ at a cooling rate of $5.6 \mathrm{~K} / \mathrm{min}$. Triplicate RVA measurements for PS had shown a high degree of reproducibility, the coefficient of variation of the peak viscosity being evaluated as only $0.19 \%$ (159.2 $\pm 0.3 \mathrm{RVU}$, mean $\pm \mathrm{SD}),{ }^{10)}$ so each measurement was subsequently taken only once.

Measurement of the absorbance of the retrograded paste. The paste after RVA measurement was held between two slide glasses separated by the thickness of one slide glass without any entrapped air bubbles, and left at $4^{\circ} \mathrm{C}$ for $24 \mathrm{~h}$ according to the previously described method. ${ }^{20)}$ After being warmed to room temperature, the absorbance at $500 \mathrm{~nm}$ of the paste was measured to evaluate the turbidity due to macroscopic aggregation of the potato starch chains during short-term retrogradation.

Measurement of the surface and interfacial tension of the OA-BOS-P solution. The surface tension of a $1 \% \mathrm{OA}-$ BOS-P solution and interfacial tension of a corn oil (J-Oil Mills, Tokyo, Japan)-1\% OA-BOS-P solution were measured at $25^{\circ} \mathrm{C}$ with a CBVP-A3 automatic surface tensiometer (Kyowa Interface Sci., Niiza, Japan) by the Wilhelmy method. ${ }^{21)}$

Conductometric titration. Conductometric titration was carried out to measure the calcium ions bound with OABOS-P and BOS-P as described previously. ${ }^{10)} \mathrm{A} \mathrm{CaCl}_{2}$ solution (1 or $2 \mathrm{mM}$ ) was added to $16 \mathrm{~mL}$ of an OA-BOS-P $(0.70 \mathrm{mg} / \mathrm{mL})$ or BOS-P $(0.71 \mathrm{mg} / \mathrm{mL})$ solution in small increments $(50 \mu \mathrm{L}$ at a time), and the conductivity was measured with an ES-12 conductometer (Horiba, Kyoto, Japan) at room temperature. The end point for conductometric titration was determined as the intersection of the linear regression curves obtained from the initial titration and from the ultimate titration.

Analytical methods. The moisture content was determined by heating a sample at $110^{\circ} \mathrm{C}$ until constant weight had been obtained. The total sugar and the reducing sugar were respectively determined by the phenol-sulfuric acid method ${ }^{22)}$ and the Somogyi ${ }^{23)}$-Nelson ${ }^{24)}$ method. $\overline{\mathrm{DP}}$ of BOS-P was determined by the ratio of the total sugar content to the reducing sugar content of the sample solution according to the previously described method, ${ }^{18)}$ after de-phosphorylating with acid phosphatase. BOS-P $(2.5 \mathrm{mg})$ dissolved in $2.5 \mathrm{~mL}$ of a $0.05 \mathrm{M}$ acetate buffer $(\mathrm{pH} 4.8)$ was treated at $27^{\circ} \mathrm{C}$ for $6 \mathrm{~h}$ with the addition of $1.0 \mathrm{~mL}$ of an acid phosphatase acetate buffer solution $(1 \mathrm{mg} / \mathrm{mL})$. The mixture was centrifuged at $1,700 \times \mathrm{G}$ for $5 \mathrm{~min}$ to obtain the supernatant for the measurement of sugars after being adjusted to $\mathrm{pH} 7.0$ with $1 \mathrm{M}$ $\mathrm{NaOH}$ and heated at $100^{\circ} \mathrm{C}$ for $5 \mathrm{~min}$. Each sugar content in the resulting supernatant was corrected by determining the amount of sugar contaminating the acid phosphatase. The amount of sucrose mono-stearate (S-1570) retained by PS was determined as follows. PS ( $2 \mathrm{~g}$ ) was dispersed in $25 \mathrm{~mL}$ of an SE solution containing $0.2 \%$ S-1570 based on the weight of PS, and the mixture was heated at $50^{\circ} \mathrm{C}$ and $75^{\circ} \mathrm{C}$ for 30 min while shaking. After centrifuging at $29,000 \times \mathrm{G}$ for $30 \mathrm{~min}$, the retained S-1570 was evaluated by measuring the fatty acid concentration of the resulting supernatant according to the previous method. ${ }^{25)}$ The oleic acid content of OA-BOS-P was next determined. The fatty acid content was then determined by gas-liquid chromatography with GC 4CM apparatus (Shimadzu, Kyoto, Japan) and a DEGS Chromosorb WAW column (GL Science, Tokyo, Japan), after the fatty acid methyl esters had been prepared from OABOS-P by methanolysis according to the method previously described. ${ }^{25}$ The phosphoric acid contents of BOS-P and OA-BOS-P were determined by wet-ashing with sulfuric acid and perchloric acid and coloring with a commercial Phospher-C test kit (Wako Pure Chemical Ind., Osaka, Japan) as described previously. ${ }^{10)}$

Statistical analysis. The Turkey-Kramer test was used to compare mean values at the $5 \%$ significance level.

\section{RESULTS AND DISCUSSION}

\section{Chemical features of BOS, BOS-P, OA-BOS and OA- BOS-P.}

$\overline{\mathrm{DP}}$ of BOS from corn starch was 11.7. The unit chainlength distribution of the debranched BOS showed that it was composed of malto-oligosaccharides from maltose (G2) to malto-dodecaose (G12); in particular, the unit chain from G2 to malto-octaose (G8) accounted for about $96 \%$ of the whole (Fig. 1). These results thus show that BOS was a branched oligosaccharide population containing different chain lengths. BOS-P was prepared by dry-heating with metaphosphoric acid. The yield was $9.4 \%$, being higher than that (about $2 \%)^{10)}$ for phosphrylated sucrose stearate that had previously been prepared in the same manner. DP of BOS-P was 12.9 , being a little higher than that of BOS. OA-BOS and OA-BOS-P were respectively prepared by oleylating BOS and BOS-P through lipase-catalyzed solid phase syn- 
thesis. The respective yields of OA-BOS and OA-BOS-P were 10.1 and $8.8 \%$. The oleic acid content of OA-BOS was $1.3 \%$ based on the sample weight, and the respective oleic acid and phosphate contents of OA-BOS-P were evaluated to be 2.9 and $6.3 \%$ based on the sample weigh.

\section{Improved interfacial ability.}

The interfacial properties of BOS-P, OA-BOS and OABOS-P were investigated in terms of the surface tension in a water-air system, and the interfacial tension in a corn oilwater system. The surface tension $(71.99 \mathrm{mN} / \mathrm{m})$ of distilled water at $25^{\circ} \mathrm{C}^{26)}$ and interfacial tension was a little decreased with BOS-P (Fig. 2). This indicates that BOS-P has a low degree of the interfacial activity. On the other hand, OABOS and OA-BOS-P exhibited markedly lower surface tension and interfacial tension than BOS-P; in particular, OABOS-P exhibited extremely low surface tension and interfacial tension (Fig. 2), being markedly lower than the values (43.1 and $13.6 \mathrm{mN} / \mathrm{m}$, respectively) of soybean lecithin. OA-BOS-P thus exhibited superior interfacial ability, probably due to the improved hydrophile-lipophile balance by oleylation, suggesting good emulsifying ability.

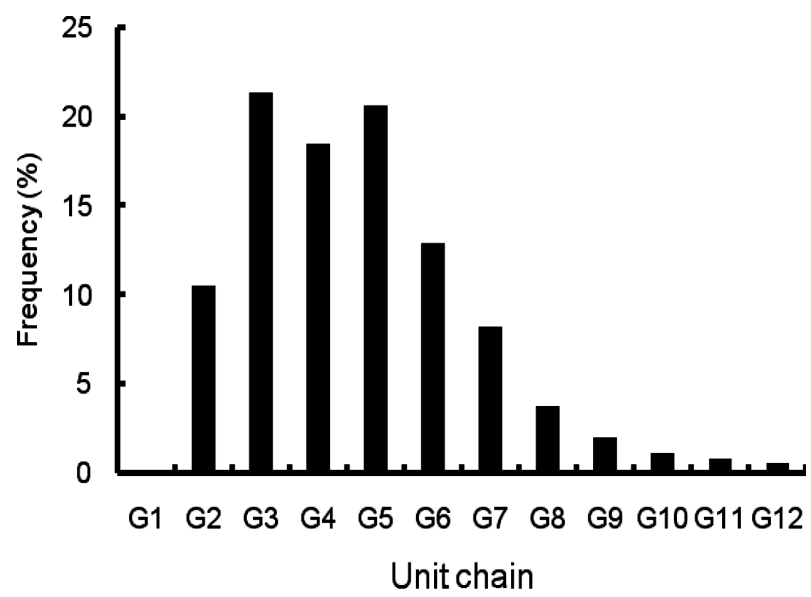

Fig. 1. Unit chain-length distribution of BOS evaluated by Dionex HPAEC.

\section{Calcium ion-binding ability.}

The $\mathrm{Ca}^{2+}$-binding ability of BOS-P and OA-BOS-P was evaluated by conductometric titration with a $\mathrm{CaCl}_{2}$ solution, and the end point of the titration was determined as the intersection between the two regression lines with high determination coefficients. ${ }^{10)}$ Consequently, although BOS showed only one straight titration curve, BOS-P and OA-BOS-P showed a curved titration trace due to binding of the added $\mathrm{Ca}^{2+}$. The respective amounts of $\mathrm{Ca}^{2+}$ bound to BOS-P and OA-BOS-P were calculated to be $10 \mathrm{mg} / \mathrm{g}$ of the sample and $5.1 \mathrm{mg} / \mathrm{g}$ of the sample, indicating that BOS-P and OA-BOS$\mathrm{P}$ acquired $\mathrm{Ca}^{2+}$-binding ability.

\section{Controlled gelatinization behavior.}

The gelatinization behavior of PS containing BOS, BOSP, OA-BOS, OA-BOS-P, and sucrose monooleiate (O-1570) was investigated by DSC and RVA. DSC showed each sample to have a significantly elevated gelatinization temperature $\left(T_{\mathrm{o}}\right)$ depending on the level of addition (Fig. 3). In the range of additional level tested, the effect of these samples on the increase in $T_{0}$ was in the order OA-BOS-P $>$ OA-BOS $>$ BOS-P $>$ BOS $>$ O-1570, probably due to effects on increased $T_{0}$ of phosphate, ${ }^{27)}$ sugar $^{28)}$ and the oleyl moiety. The enthalpy change $\left(\Delta H_{1}\right)$ decreased by adding each sample (Fig. 3); comparing the results with $10 \%$ addition, the $\Delta H_{1}$ reducing effect was in the order OA-BOS-P $\approx 0-1750>\mathrm{OA}-$ $\mathrm{BOS}>\mathrm{BOS}-\mathrm{P} \approx \mathrm{BOS}$. It is well-known that DSC detects the endothermic heat flow caused by phase transition of the ordered structure during gelatinization, being similar to that for the denaturation of protein. The thermal transition of protein has been evaluated by the entropy change $(\Delta S)$ derived by assuming that the protein denaturation can be regarded as a phase transition of the melting type, and that the free energy change $(\Delta G)$ at the transition point is zero. ${ }^{29,30)}$ In order to investigate the effect of OA-BOS-P on the gelatinization behavior, this approach was applied by using the following equation that expresses the Gibbs free-energy change: $\Delta G=$ $\Delta H-T \Delta S=0, \Delta S=\Delta H / T$, where $T$ is the absolute temperature, and $\Delta H$ and $\Delta S$ are calculated on the basis of the glu-
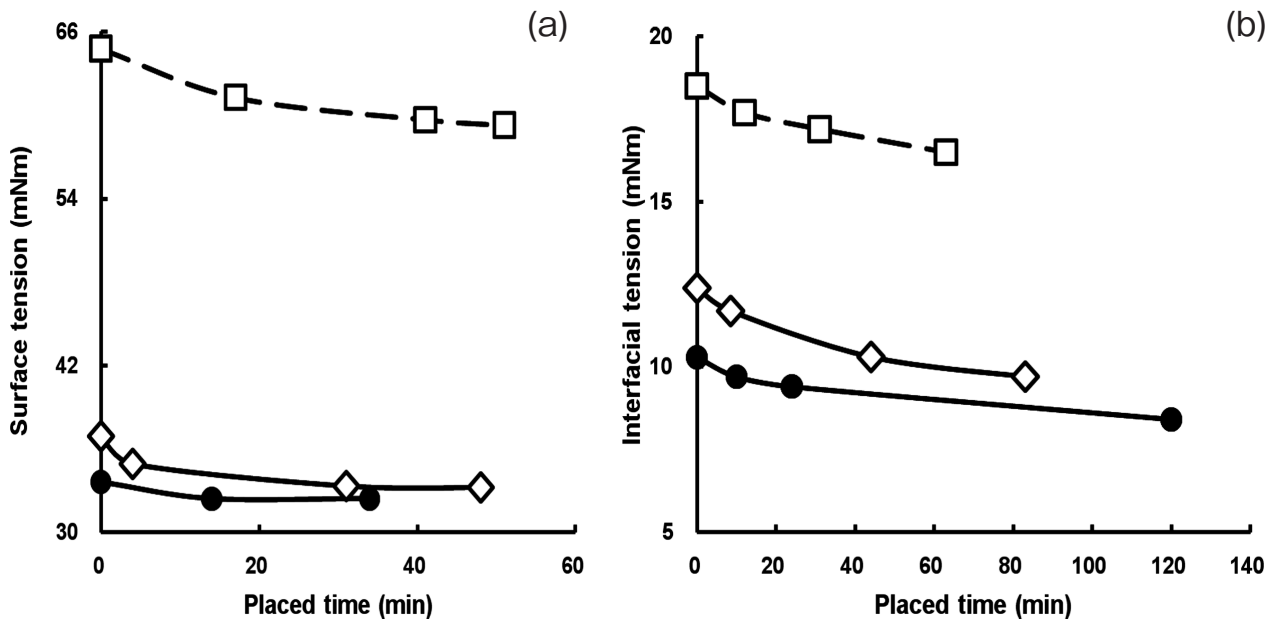

Fig. 2. Comparative effect of OA-BOS-P on (a) the surface tension in a water-air system and (b) the interfacial tension in a watercorn oil system with those of the related samples.

๑, OA-BOS-P; $\diamond$, OA-BOS; $\square$, BOS-P 

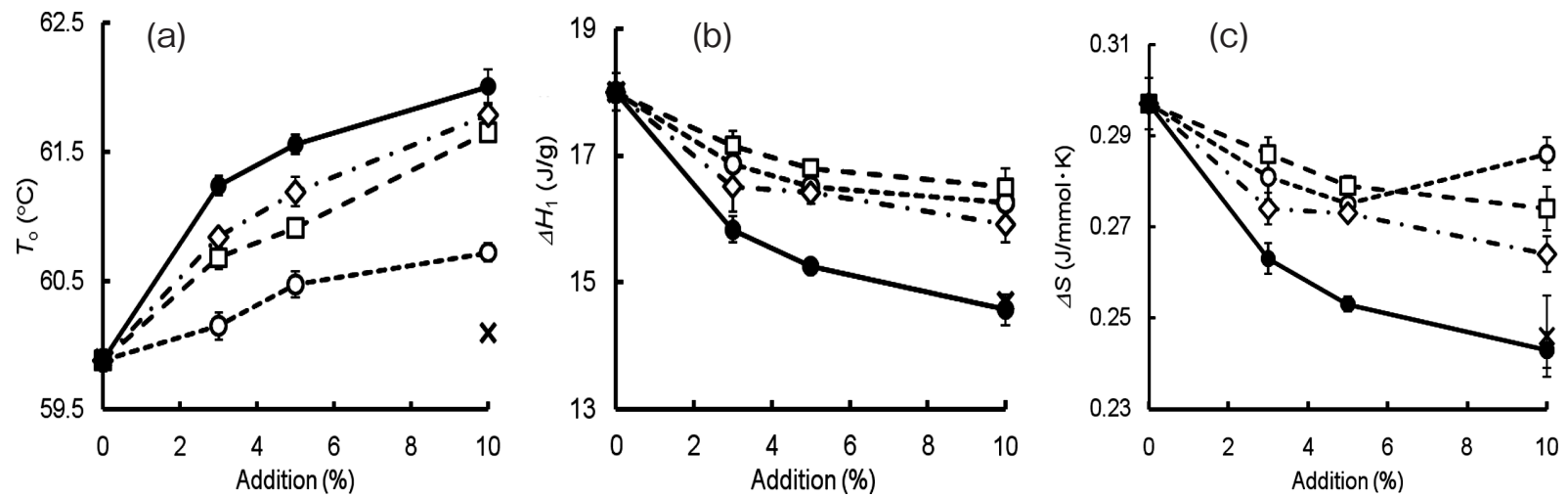

Fig. 3. Comparative effects of OA-BOS-P on (a) the gelatinization temperature $\left(T_{0}\right)$, (b) enthalpy change $\left(\Delta H_{1}\right)$ and (c) entropy change $(\Delta S)$ of potato starch evaluated by DSC with those of the related samples.

, OA-BOS-P; $\square$, BOS-P; $\diamond$, OA-BOS; $\bigcirc$, BOS; × , sucrose mono-oleiate (O-1570, Mitsubishi Kagaku Foods Co., Tokyo, Japan). The symbol and error bar respectively show the mean value and standard deviation of five measurements. Any omitted error bars were too close to show.

cose residue of starch. Since $\Delta S$ can be interpreted as an index of the degree of disorder of the ordered structure during the thermal transition of starch, a starch state showing a smaller $\Delta S$ value could be interpreted as that having higher resistance to disordering of the starch structure due to gelatinization. The $\Delta S$ value significantly decreased in each sample depending on the level of addition (Fig. 3), this reduction being in the order OA-BOS-P $\geq 0-1570>$ OA-BOS $>$ BOS$\mathrm{P} \approx \mathrm{BOS}$. It is thus suggested that OA-BOS-P could most markedly elevate the rigidity (resistance to disordering) of the starch structure, presumably contributing to inhibiting rupture of the swollen starch granules.

In order to presume the probable reason for the large effect of OA-BOS-P, the amount of S-1570 retained by PS was determined as a substitute for OA-BOS-P, because the saturated fatty acid would have higher affinity to the starch chains than the unsaturated fatty acid. ${ }^{31)}$ The results showed that 25 and $88 \%$ of the added S-1570 were respectively retained with PS at 50 and $75^{\circ} \mathrm{C}$ (corresponding to $3.4 \mathrm{~S}-1570$ and $6.1 \mathrm{~S}-1570 \mathrm{~mol} / 1,000$ glucose residues of PS). This suggests that OA-BOS-P would be fairly well retained by complexion with the starch chain via the fatty acid moiety, and that the retained OA-BOS-P could inhibit the hydration of PS. In fact, a separate experiment showed that PS containing OA-BOS-P appeared reversibly as a small endothermic peak at about $103^{\circ} \mathrm{C}$ (peak temperature; data not shown), corresponding to the melting endothermic peak of a starch-fatty acid complex. ${ }^{10,15)}$ We have previously reported that a charged amino acid exhibited higher binding strength to the starch chains through electrostatic interaction than amino acids with zero net charge. ${ }^{32)}$ This suggests that OA-BOS-P could well bind to starch chains via the phosphate moiety with a negative charge. The retention of OA-BOS-P to starch granules and binding to the starch chains presumably resulted in the increased rigidity of the starch structure.

Each sample influenced the pasting behavior of PS. The peak viscosity (PV) and breakdown (BD) decreased with BOS, BOS-P, OA-BOS, OA-BOS-P and O-1750 depending on the level of addition (Fig. 4) without any change in the pasting temperature, and the decrease in PV with $1 \%$ addition was in the order OA-BOS-P $>$ OA-BOS $\geq$ BOS-P $>$ $\mathrm{BOS} \geq \mathrm{O}-1750$. The relationship between the $\mathrm{PV}$ value $(Y)$ or BD value $(Y)$ with $1 \%$ addition and the $\Delta S$ value with $3 \%$ addition $(X)$ could be expressed by the following linear re- gression equations: $Y=1.22 \times 10^{4} X-3150(R=0.842)$ for $\mathrm{PV} / \Delta S$, and $Y=9,230 X-2,390(R=0.826)$ for $\mathrm{BD} / \Delta S$. The relatively high correlation coefficients suggest that the swelling of starch granules could be inhibited by the increased rigidity of the starch structure by the retained OA-BOS-P. These results therefore indicate that OA-BOS-P could more markedly improve the gelatinization behavior than the other samples could, in terms of the increased gelatinization temperature and reduced viscosity of the paste due to the inhibition of swelling of the starch granules, suggesting a strongly inhibiting effect on the development of the sticky and pasty texture.

\section{Controlled retrogradation behavior.}

The effects of OA-BOS-P and related samples on the initial and short-term retrogradation behavior were respectively evaluated by the setback (SB) during cooling to $50^{\circ} \mathrm{C}$ in the RVA measurements and by the turbidity, that is, the increased absorbance at $500 \mathrm{~nm}(\triangle \mathrm{Abs})$ of the paste retrograded at $4^{\circ} \mathrm{C}$ for $24 \mathrm{~h}$ after RVA. Each sample lowered SB depending on the level of addition, except for $0.5 \%$ BOS-P addition elevating it to some degree (Fig. 4); the decrease with $1 \%$ addition was in the order OA-BOS-P $>$ OA-BOS $>$ BOS $>$ BOS-P $>$ $\mathrm{O}-1750$. The strongly inhibited initial retrogradation by OABOS-P and OA-BOS was probably caused by an effective inhibition of the entanglement resulting from the starch-OABOS-P complexes formed by the oleyl moiety as described above. In addition, since O-1570 showed only low inhibition, it seems that the bulky structure of BOS and the electrostatic repulsion of the phosphate moiety contributed to the greater inhibition by OA-BOS-P and OA-BOS. The relationship between the SB value $(Y)$ with $1 \%$ addition and the $\Delta S$ value with $3 \%$ addition $(X)$ could be expressed by the following linear regression equation: $Y=761 X-199(R=$ 0.972 ). It is thus suggested that the rigidity of the starch chains controlled with OA-BOS-P and related samples could contribute to inhibiting the initial retrogradation. The initial retrogradation inhibited by BOS-P may have been due to the phosphate moiety with the negative charge, because negatively charged glutamic acid was strongly attached to the starch chains via electrostatic interaction and thus reduced SB. ${ }^{33)}$ The short-term retrogradation appeared to follow unusual behavior. BOS-P and OA-BOS elevated the turbidity of the starch paste more than the control did, although OA- 


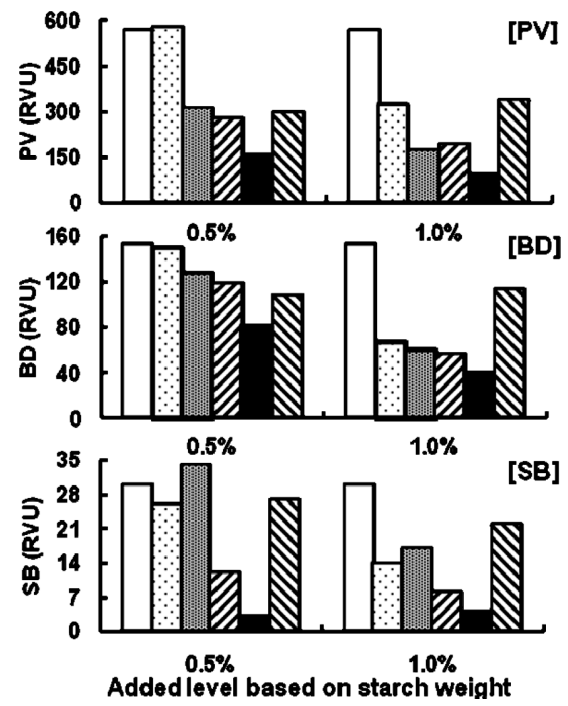

Fig. 4. Comparative effects of OA-BOS-P on the peak viscosity $(\mathrm{PV})$, breakdown (BD) and setback (SB) of potato starch evaluated by RVA with those of the related samples.

OA-BOS-P; $\square$, BOS-P; שIA, OA-BOS; $\because$, BOS; $\mathbf{M}$, sucrose mono-oleiate (O-1570); $\square$, control.

BOS-P and BOS exhibited reduced turbidity (Fig. 5). This suggests that BOS-P and OA-BOS promoted the short-term aggregation of starch, in spite of inhibiting the initial entanglement of the starch chains, and that OA-BOS-P and BOS inhibited aggregation, corresponding well with the inhibited initial entanglement of the starch chains. It is thus concluded that OA-BOS-P could inhibit the initial and short-term retrogradation as indicated by the inhibited entanglement and aggregation of the starch chains, whereas BOS-P accelerated this effect.

The effect of OA-BOS-P and related samples on the relatively long-term retrogradation in terms of the development of an ordered structure was examined by DSC, using samples preserved at $4^{\circ} \mathrm{C}$ for 7 days after the first DSC run. BOS-P significantly elevated the re-gelatinization enthalpy $\left(\Delta H_{2}\right)$ with $10 \%$ addition, although there was no significant difference below $5 \%$ addition (Fig. 6). This indicates that BOS-P increased the development of an ordered structure corresponding to the increased aggregation of starch chains during short-term retrogradation. However, the other samples substantially reduced $\Delta H_{2}$; in particular, OA-BOS-P significantly reduced $\Delta H_{2}$ depending on the addition level, probably due to forming a complex with a starch chain via the olelyl moiety and due to electrostatic repulsion via the phosphate moiety. Since the relationship between the $\Delta H_{2}$ value $(Y)$ and $\Delta S$ value $(X)$, excepting the results for BOS-P, could be expressed by the following linear regression equation: $Y=29.8 X+0.7(R=0.815)$, it is suggested that the rigidity of starch structure controlled by OA-BOS-P and related samples, excepting BOS-P, could have inhibited the relatively long-term retrogradation in terms of the reduced development of an ordered structure. This result implicates OA-BOS-P in controlling the gelatinization and retrogradation behavior of PS as a novel modifier.

The comparative effects of OA-BOS-P on the thermal behavior of PS with those of BOS, BOS-P, OA-BOS and $\mathrm{O}-1570$ are summarized in respect to the rigidity $(\Delta S)$ during gelatinization with $3 \%$ addition, pasting properties (PV and

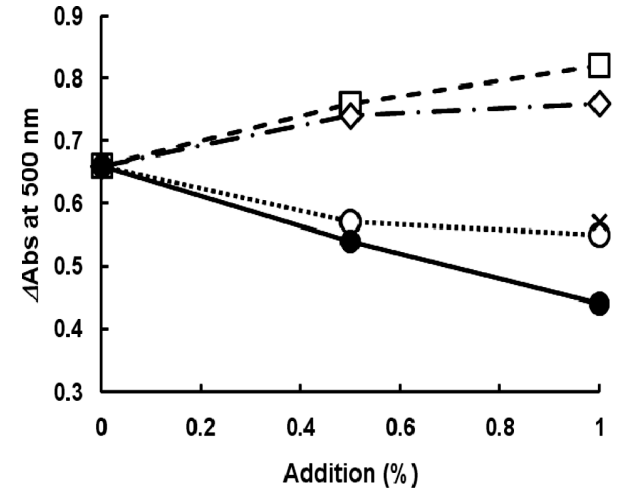

Fig. 5. Comparative effect of OA-BOS-P on the increased absorbance $(\triangle \mathrm{Abs})$ at $500 \mathrm{~nm}$ of the potato starch paste retrograded at $4^{\circ} \mathrm{C}$ for $24 \mathrm{~h}$ after RVA with that of the related samples.

๑, OA-BOS-P; $\bigcirc$, BOS; $\square$, BOS-P; $\diamond$, OA-BOS; $\times$, O-1570.

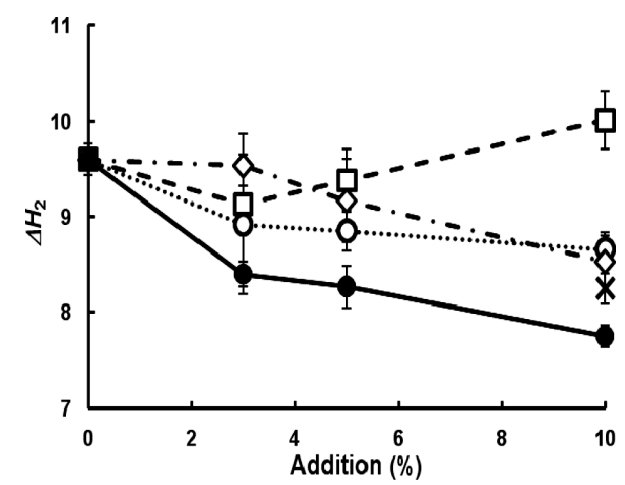

Fig. 6. Comparative effect of OA-BOS-P on the re-gelatinization enthalpy $\left(\Delta H_{2}\right)$ of starch paste preserved at $4^{\circ} \mathrm{C}$ for 7 days after the first DSC measurement with that of the related samples.

๑, OA-BOS-P; $\bigcirc$, BOS; $\square$, BOS-P; $\diamond$, OA-BOS; $\times$, O-1570. Each value is expressed as the mean and standard deviation of three measurements. Any omitted error bars were too close to show.

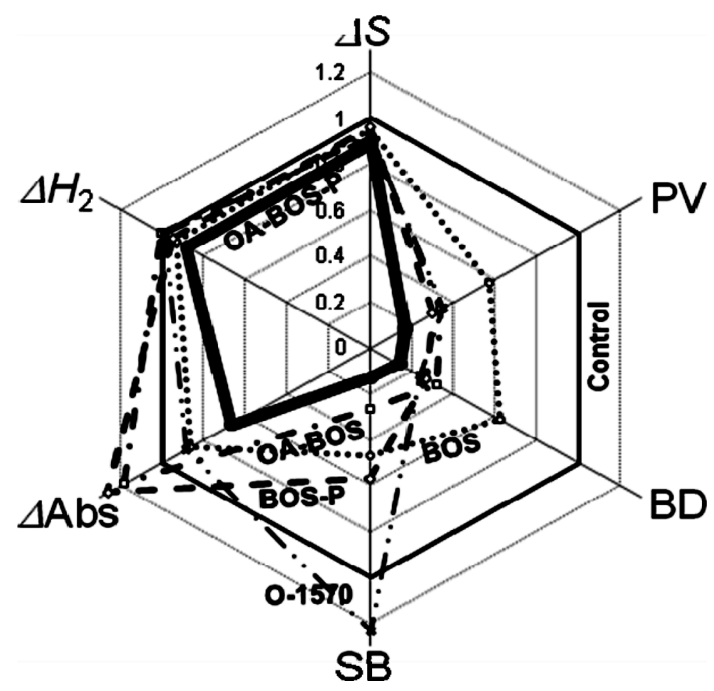

Fig. 7. Comparative effects of OA-BOS-P on the thermal properties of potato starch with those of the related samples.

Each characteristic parameter for gelatinization and retrogradation is expressed as a relative value to that of PS. $\Delta S$, entropy change of gelatinization; PV, peak viscosity; BD, break down; SB, setback; $\triangle \mathrm{Abs}$, increased absorbance at $500 \mathrm{~nm}$ of the sample retrograded at $4^{\circ} \mathrm{C}$ for $24 \mathrm{~h} ; \Delta H_{2}$, enthalpy change of the sample retrograded at $4^{\circ} \mathrm{C}$ for 7 days. O-1570; - OA-BOS-P. The data for $\Delta S$ and $\Delta H_{2}$ are derived from the values with $3 \%$ addition, and those for $\mathrm{PV}, \mathrm{BD}, \mathrm{SB}$ and $\Delta \mathrm{Abs}$ are from the values with $1 \%$ addition. 
BD) with $1 \%$ addition, and the retrogradation behavior [SB, turbidity $(\triangle \mathrm{Abs})$, and development of the ordered structure $\left(\Delta H_{2}\right)$ ] compared to the results for the control (Fig. 7). OABOS-P substantially increased the rigidity, as indicated by the low $\Delta S$ value in comparison with the other samples. Each sample, particularly OA-BOS-P, reduced the PV and BD values. Furthermore, OA-BOS-P strongly inhibited retrogradation over 7 days at $4^{\circ} \mathrm{C}$ in respect to the entanglement and aggregation of the starch chains respectively indicated by the low SB and $\triangle \mathrm{Abs}$ values. In contrast, the other samples resulted in either relatively low inhibition or accelerated retrogradation, as indicated by higher $\mathrm{SB}$ and $\triangle \mathrm{Abs}$ values than the control. It is thus concluded that OA-BOS-P would be a useful multi-functional material with novel interfacial ability, calcium ion-binding ability and starchy food-modifying ability.

\section{ACKNOWLEDGMENTS}

We are grateful to Associate Professor T. Hamanishi of Kyoritsu Women's University for measuring RVA, and to Mr. T. Itaya of Hokuren Research Institute for providing large-granule potato starch.

\section{REFERENCES}

1 ) T. Watanabe: Sucrose fatty acid esters - past, present and future. Foods Food Ingredients J. Japan, 180, 18-25 (1999) (in Japanese).

2 ) K. Yamamoto, H. Kumagai, T. Sakiyama, M. Song and T. Yano: Inhibitory activity of alginates against the formation of calcium phosphate. Biosci. Biotechnol. Biochem., 56, 90-93 (1992).

3 ) Y-S. Lee, T. Noguchi and H. Naito: Phosphopeptides and soluble calcium in the small intestine of rats given a casein diet. $B r . J$. Nutr., 43, 457-467 (1980).

4 ) H. Kamasaka, M. Uchida, K. Kusaka, K. Yoshikawa, K. Yamamoto, S. Okada and T. Ichikawa: Inhibitory effect of phosphorylated oligosaccharides prepared from potato starch on the formation of calcium phosphate. Biosci. Biotechnol. Biochem., 59, 1412-1416 (1995).

5 ) K. To-o, H. Kamasaka, T. Nishimura, T. Kuriki, S. Saeki and Y. Nakabou: Absorbability of calcium-bound phosphoryl oligosaccharides in comparison with that from various calcium compounds in the rat ligated jejunum loop. Biosci. Biotechnol. Biochem., 67, 1713-1718 (2003).

6 ) G. Williams and J.D. Sallis:. Structure-activity relationship of inhibitors of hydoxyapatite formation. Biochem. J., 184, 181-184 (1979).

7 ) F. Nakazawa, J. Takahashi and M. Takada: Interaction between potato starch and sucrose-lipid monoesters studied by differential scanning calorimetry. Biosci. Biotechnol. Biochem., 62, 248-252 (1998).

8 ) N. Krog and B. Nybo-Jensen: Interaction of monoglycerides in different physical states with amylose and their anti-firming effects in breads. J. Food Technol., 5, 77-87 (1970).

9 ) M. Yamashita, H. Adachi, T. Nakamura, N. Tokuriki, H. Taniguchi and M. Hisamatsu: Effect of soy lysophospholipid on rheological properties of wheat starch gel. J. Appl. Glycosci., 48, 271278 (2001).

10) Y. Yamagishi, M. Hattori, T. Yoshida and K. Takahashi: Improvement of the functional properties of sucrose stearate by phosphorylation. J. Agric. Food Chem., 52, 8039-8045 (2004).

11) K. Katsuta, M. Miura and A. Nishimura: Kinetic treatment for rheological properties and effects of saccharides on retrogradation of rice starch gels. Food Hydrocolloids, 6, 187-198 (1992).

12) K. Katsuta, A. Nishimura and M. Miura: Effect of saccharides on stabilities of rice gels. 1. Mono- and disaccharides. Food Hydrocolloids, 6, 387-398 (1992).
13) M. Ogino, R. Tanaka, M. Hattori, T. Yoshida, Y. Yokote and K. Takahashi: Interfacial behavior of fatty-acylated sericin prepared by lipase-catalyzed solid-phase synthesis. J. Food. Chem., 70, 66-75 (2006).

14) M.C. Tang and L.Copeland: Analysis of complexes between lipids and wheat starch. Carbohydr. Polym., 67, 80-85 (2007).

15) Z. Zhou, K. Robards, S. Helliwell and C. Blanchard: Effect of the addition of fatty acids on rice starch properties. Food Res. Int., 40, 209-214 (2007).

16) E. Tarelli and S.F. Wheeler: Drying from phosphate-buffered solutions can result in the phosphorylation of primary and secondary alcohol groups of saccharides, hydroxlyated amino acids, proteins and glycoproteins. Anal. Biochem., 222, 196-201 (1994).

17) S. Sakauchi, M. Hattori, T. Yoshida, T. Yagishita, K. Ito, S. Akemitsu and K. Takahashi: Thermal behavior of potato starch and water-vaporization behavior of its paste controlled with amino acid and peptide-rich food materials. J. Food Sci., 74, C177183 (2009)

18) K. Takahashi, H. Kondo, H. Kuroiwa, Y. Yokote and M. Hattori: Reversible thermal transition of soluble branched chains from slightly acid-treated potato starch. Biosci. Biotechnol. Biochem. 64, 1365-1372 (2000).

19) K. Takahashi, K. Shirai and K. Wada: Melting behavior of gels prepared from isolated subunits of collagen. J. Food Sci., 53, 1920-1921 (1988).

20) S. Katayose, K. Kegai, M. Nishimura, N.A. bt. Daud, M. Hattori, T. Yoshida, Y. Ishii and K. Takahashi: Starch- $\varepsilon$-poly(L-lysine)fatty acylated saccharide and $\varepsilon$-poly(L-lysine)-fatty acylated saccharide conjugates exhibit emulsifying ability, antibacterial activity, and controlling ability of thermal behavior of potato starch. $J$. Appl. Glycosci., 54, 173-180 (2007).

21) L. Wilhelmy: Ueber die abhängigkeit der capillartäts-consten des alcohols von substanz und des benetzten fasten körpers. Ann. Physik, 12, 177-217 (1863).

22) M. Dubois, K.A. Gilles, J.K. Hamilton, P.A. Rebers and F. Smith: Colorimetric method for determination of sugars and related substances. Anal. Chem., 28, 350-356 (1956).

23) M. Somogyi: Notes on sugar determination. J. Biol. Chem., 195, 19-23 (1952).

24) N. Nelson: A photometric adaptation of Somogyi method for the determination of glucose. J. Biol. Chem., 153, 357-380 (1944).

25) K. Takahashi X-F. Lou, Y. Ishii and M. Hattori: Lysozyme-glucose stearic acid monoester conjugate formed through the Maillard reaction as an antibacterial emulsifier. J. Agric. Food Chem., 48, 2044-2049 (2000).

26) N.B. Vargaftik, B.N. Volkov and L.D. Voljak: International tables of the surface tension of water. J. Phys. Chem. Ref. Data, 12, 817 (1983).

27) K. Takahashi, K. Shirai, K. Wada and A. Kawamura: Effects of salts and saccharides on the gelatinization of starch. J. Jpn. Soc. Starch Sci., 27, 22-27 (1980) (in Japanese).

28) A. Beleia, R.A. Miller and R.C. Hoseny: Starch gelatinization in sugar solutions. Starch/Stärke, 48, 259-262 (1996).

29) P.L. Privalov and E.I. Tikutopulo: Thermal conformational transformation of tropocollagen I. Calorimetric study. Biopolymers, 9 , 127-139 (1969).

30) D. Eagland: The helix-coil transition of native collagen and soluble tropocollagen and the development of the collagen fold by gelatins in aqueous solution. in Water A Comprehensive Treaties, vol. 4, F. Franks, ed., Plenum Press, New York, pp. 363-370 (1975).

31) M.C. Tang and L. Copeland: Analysis of complexes lipids and wheat starch. Carbohydr. Polym., 67, 80-85 (2007).

32) A. Ito, M. Hattori, T. Yoshida, A. Watanabe, R. Sato and K. Takahashi: Regulatory effect of amino acids on the pasting behavior of potato starch is attributable to its binding to the starch chain. $J$. Agric. Food Chem., 54, 10191-10196 (2006).

33) A. Ito, M. Hattori, T. Yoshida and K. Takahashi: Contribution of the net charge to the regulatory effects of amino acids and $\varepsilon$-poly (L-lysine) on the gelatinization behavior of potato starch granules. Biosci. Biotechnol. Biochem., 70, 76-85 (2006). 